\title{
FROM THE EXTRAORDINARY NATURE OF THE GREAT POMPEII PROJECT TO PLANNED CONSERVATION
}

\author{
A. Mauro \\ Funzionario Architetto, Parco Archeologico di Pompei, 80045 Pompei, Napoli Italy, annamaria.mauro@beniculturali.it
}

KEY WORDS: Knowledge, Conservation,Maintenance, Resilience, Technology

\begin{abstract}
:
The Archaeological Park of Pompeii promotes the most advanced methodologies in preservation, conservation, research, and the promotion of public use of the site. The Great Project Pompeii (GPP), as is evident, has intervened 'strategically' on the entire site: on the one hand stabilising the Regiones, on the other hand mitigating the hydrogeological risk of the plateaus and securing the excavation fronts; in addition the GPP has improved accessibility levels. Coping with environmental risks (seismic, volcanic and hydrogeological) is one of the main challenges of the Archaeological Park. First, seismic and volcanic phenomena constitute a major issue in the Pompeii area, embodied by the dormant Mt. Vesuvius. Second, the marked difference in height between the level of the ancient town and that of the unexcavated area, spanning about 22 ha, exerts a strong pressure over the ancient structures and may imply a serious danger of landslides in case of hazard events (seismic and volcanic phenomena or heavy rainfall). These dangers seriously jeopardise the protection of ancient structures and wall-paintings, with subsequent risks to the world cultural heritage site. For this reason, an urgent program of conservation, maintenance, and restoration has been undertaken as part of the Great Pompeii Project. The computerisation of archaeological data is among the most current topics of interest for the management of Cultural Heritage. The Information System (GPP-IS), created with the Plan of the Knowledge of the Great Pompeii Project (GPP), has allowed the creation of a unique knowledge tool for the entire city of Pompeii.
\end{abstract}

\section{THE ROLE OF THE GPP-IS, CHALLENGES IN THE DEVELOPMENT OF THE SYSTEM AND IN ITS EVERYDAY USE, AS WELL, AS THE USE OF ITS DECISION- MAKING PROCESS.}

The Archaeological Park of Pompeii promotes the most advanced methodologies in preservation, conservation, research, and the promotion of public use of the site. The Great Project Pompeii (GPP), as is evident, has intervened 'strategically' on the entire site: on the one hand stabilising the Regiones, on the other hand mitigating the hydrogeological risk of the plateaus and securing the excavation fronts; in addition the GPP has improved accessibility levels. Coping with environmental risks (seismic, volcanic and hydrogeological) is one of the main challenges of the Archaeological Park. First, seismic and volcanic phenomena constitute a major issue in the Pompeii area, embodied by the dormant Mt. Vesuvius. Second, the marked difference in height between the level of the ancient town and that of the unexcavated area, spanning about 22 ha, exerts a strong pressure over the ancient structures and may imply a serious danger of landslides in case of hazard events (seismic and volcanic phenomena or heavy rainfall). These dangers seriously jeopardise the protection of ancient structures and wall-paintings, with subsequent risks to the world cultural heritage site. For this reason, an urgent program of conservation, maintenance, and restoration has been undertaken as part of the Great Pompeii Project. The computerisation of archaeological data is among the most current topics of interest for the management of Cultural Heritage. The Information System (GPP-IS), created with the Plan of the Knowledge of the Great Pompeii Project (GPP), has allowed the creation of a unique knowledge tool for the entire city of Pompeii. This system should be the reference point for all subsequent interventions of maintenance, restoration and archaeological investigation. It has been possible, for the first time, to have an instrument capable of providing standardised and analytical data for all the buildings and the infrastructure of the ancient city, as well of providing constant updates of the evaluation parameters for the conservational status of individual assets, and of increasing the information assets according to already defined criteria.

The primary objective of the GPP-IS is to create a system which, based on a relational data base linked to a georeferenced map, would support the management of the 'scheduled maintenance' process. The implementation of the planned maintenance process aims to prevent degradation and to monitor the risk conditions, favouring ordinary maintenance activities over post eventum restoration activities that intervene on an already-altered situation which has been irreparably compromised by the loss of elements and historical testimonies. The Information System (GPP-IS) is a web-based service provided by a cloud infrastructure. The System constitutes a unique and exhaustive container capable of understanding and managing all the historical scientific knowledge accumulated over the years regarding the archaeological area. It is therefore possible to preserve, and to interconnect, all different documents. The system provides for the registration, via the appropriate form, of each intervention carried out on a specific asset (or part of it). This allows a timely report of all the interventions carried out over the time, to be able to check, during the inspection phase, the effectiveness and durability of the interventions, thus allowing the preparation a manual of the interventions which are more suitable to counteract the different forms of degradation. The GPP-IS provides a system for defining the knowledge of each element at the Pompeii archaeological site, allowing the construction of geographical, statistical and documentary information relating to each object in the hierarchy, envisaged by the scheduling system. At an 'urban' scale site like Pompeii, where the planning of maintenance activities must face conservational urgencies on a daily basis, it is necessary to operate utilising a strategy of the 
progressive definition of interventions. The Great Pompeii Project has represented the beginning of a general rethinking of the management and use $\&$ access models of the archaeological site. In this way, a path has been laid out, aimed at identifying the methodologies and resources necessary to proceed with the gradual transition from the extraordinary nature of the GPP to a continuous and planned conservation operation. The role of Pompeii as a crucial historical site and a privileged laboratory of multi- and interdisciplinary advanced research opens up a number of opportunities for the multifaceted range of participants. The ongoing effort to manage environmental risks - seismic, volcanic and hydrogeological - of the area will strengthen the resilience of the site and the communities connected with it.

The Great Pompeii Project Information System is an instrument both for understanding and managing the Ancient City of Pompeii.

The current information base, upon which the entire cognitive system of the Ancient City of Pompeii is based, consists of several levels of documentation which have been acquired over an extended period of time and which, merged with the Pompeii IS, are appropriately structured and standardised. Naturally, the wealth of cognitive heritage gathered over the years on the archaeological area, beginning with the earliest discoveries, is immense and exceedingly heterogenous. This Information System also holds records of all reported interventions and those concerning operations of planned conservation, inspections, site usage across the entire Archaeological Park as well as the monitoring of worksites, which keeps track of all past and ongoing operations in a designated section. As a result of this unique situation, and of the unprecedented extent of the area, we are proceeding with a high degree of systematisation, in order to achieve a standardised result which, when fully operational (IS), allows the coherent and uniform collection of all data relating to the Archaeological Park of Pompeii, not only what is already available but also what will be produced as part of the Plan of Knowledge, and during periodic monitoring. As a result we have a tool which is able to supply both standardised and analytical data for every single structure or element of infrastructure of the ancient city, as well as the ability to constantly update the evaluation parameters of the conservational state of individual cultural assets, and therefore refine the wealth of information available. In this unique and complex archaeological context, the decision to implement an Information System, using its own GIS procedures and many other forms of geomatic technology, has allowed us to hone the correspondence between the cartographic information, or the 'trace' of every single element of the ancient city, and alphanumeric information, with the end result that 'a-spatial' information is linked, allowing us to resolve other and more complex problems of data organisation. When discussing (Territorial) Information Systems, problems are often caused by confusing the acronym TIS with the Italian translation of GIS, which is none other than one of the tools used (today) to implement a TIS. However, it is not so. Territorial Information Systems are tools of analysis and decision-making, arranged in a system of men and procedures, for the gathering and distribution of data, as part of a structure which is able to supply information, aimed at the rational exploitation of resources. The 'Information System' is independent of any automation. Information Systems have indeed existed for centuries, and been developed by organisations whose raison d'être is the management of information, independently of the utilised medium (paper, electronic etc.). The GISes (Geographical Information Systems) form the electronic component of the TIS. They are containers of (territorial) information which, when properly managed, lead to the creation of thematic maps related to many aspects of the area, including: cadastral maps, geoenvironmental maps, archaeological maps and regional technical maps etc. In reality, the term TIS and GIS are erroneously considered synonyms in commonly used terminology, and used interchangeably. Furthermore, additional confusion ensues with the term 'informative' and 'informatic'. The Information System, in the meaning which is summarised here, has always existed - there was a system which informed 'of something', previously with clay tablets. The added value comes from the term 'informatic', in which we have the contraction of the meaning of 'information + automatic' into 'informatic', which modern technology today allows us1. Strictly speaking therefore the Information System, making extensive use of computerised geomatic technologies, assumes the connotation of 'informatic'. Having said that, it should be specified that the Pompeii IS is not a mere 'digitalisation' of the existing wealth of information which exists on Pompeii. In other words, even if there were an enormous initial phase of 'digitalisation' of pre-existing data, we have since moved on to the management of the entire wealth of information (now), with the aid of sophisticated 'digital' specialist procedures. The fully operational system is made available to all those who are involved in various capacities in management and conservation at the Archaeological Site of Pompeii. The construction of the Pompeii IS has been entirely web-oriented 2 - i.e. the results are easily reproduced on the web with shared and universal protocols - and it is based on Open Source technologies which have now reached a remarkable level of maturity. It's application modules and plugins have been developed in Java, which is itself found all over the world. In order to use it one does not, in practice, require any installation on one's own computer - it is enough to connect to the Pompeii IS server using one's credentials, to access the management and consultation areas, with levels of accessibility assigned by the administrator. In the briefest of summaries, the features of the main application modules which were created as part of the GPP-IS are:

\section{Editing and consultation area (personal data and geographic)}

${ }^{1}$ By its very nature the GIS, based on georeferenced areas, is typically used for geographical, and thus cartographical, processing including land and environmental management etc.

The notable difference between a conventional and archaeological GIS lies precisely in their objectives: a public service, or even a company, creates an Information System solely to manage current situations, or to predict future scenarios in order to take decisions; in archaeology the TIS (with the aid of the GIS), as well as for cataloguing, is useful to reconstruct and explain past situations, adding the temporal component to the spatial context.

2 Geographic Information Systems (GIS) published on the web are called WebGISes. A WebGIS is thus the web extension of applications created and developed to manage numeric cartography. A WebGIS project is distinguished from a GIS project by its specific aims of communication and of sharing information with other users. With WebGISes, GIS applications which were traditionally developed for stand-alone users or in LAN environments can be implemented on web servers, allowing interaction, via the internet, with the map and data associated with it. The most well-known examples of WebGISes are web applications for map localisation, street maps or online atlases. WebGIS applications can be used with Internet browsers, at times with the installation of specific plugins, or by means of separate software such as Google Earth.

Other typical examples of GIS applications which have been published in a WebGIS version are the Territorial Information Systems (TIS) of the Regions and various Comuni: these make environmental, urbanistic and territorial information accessible, offering navigation on a cartographic basis for each. 
Inspection area

Planned Conservation area

Worksite Management area

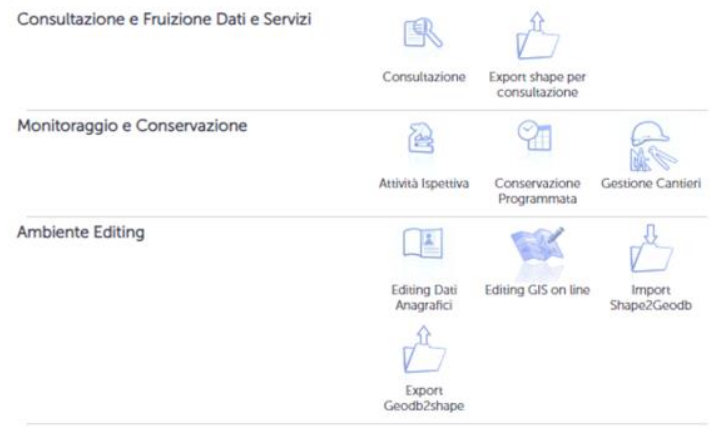

Figure 1. application modules

\subsection{Editing and Consultation Area}

This provides all the management and consultation tools for the registry data and geodata of entities which make up the archaeological site.

The objects managed in the system range from the administrative subdivision (Site, Regione and Insula) describing the practicability and numbering system - to the inhabited areas (with cadastral units, rooms, ancient furnishings, surfaces and surface decorations), and finally the description of modern furnishings, safeguarding elements and technological networks. It is intended to enrich the first set of objects with the elements which describe the archaeological excavation: from the specimen to the stratigraphic unit.

\subsection{Inspection Area}

This module provides all the management and monitoring tools required for the process of gathering data related to diagnoses, documentation, investigations and the mapping of degradation and of materials which need to be created as part of the Inspection Campaigns. As part of the Plan of Knowledge, this module has made the archiving of over 59,000 degradation profiles, by operators of the contracting companies, possible.

The participants who manage this module are: Procedural Managers, Implementation Managers, Coordinators and Operators of the contracted companies. The users intervene in the procedural flow according to the profile assigned to them, during the different phases of execution in the Inspection Campaigns.

\subsection{Planned Maintenance Area}

This provides the tools to analyse the data acquired in the inspection campaigns, for the purpose of planning the conservational interventions, as well as ordinary and extraordinary maintenance.

The solution is made available to Authorising Officials, and Planners for the Project. Here too, users intervene according to their assigned profile, in the procedural flow in the different
Planning phases (Scenario Analysis) and Planning (Preliminary, Final and Executive).

\subsection{Intervention Planning}

For each intervention, a workflow guides users to a Planner during the Preliminary and Definitive planning phases, and guarantees a validation process for the documentation produced by the Authorising Official.

\subsection{Worksite Management Area}

This provides tools for the management and monitoring of worksites, with particular attention to the impact that interventions can have on the usage of the Archaeological Site. For this purpose, a GIS function allows the definition of worksite areas, in order to identify the archaeological areas involved and to carry out spatiotemporal analysis on the coexistence of worksite areas. The solution is used by those participants involved in the intervention execution: Officials in charge of the Intervention Project and associated Planners. Users intervene in the intervention execution process according to the profile assigned to them.

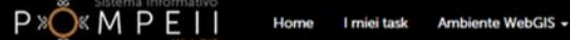

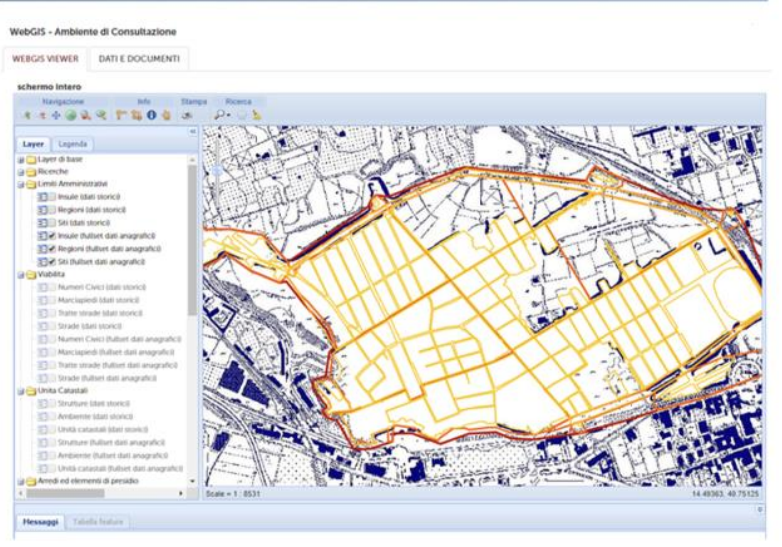

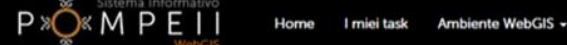

Figure 2. worksite area

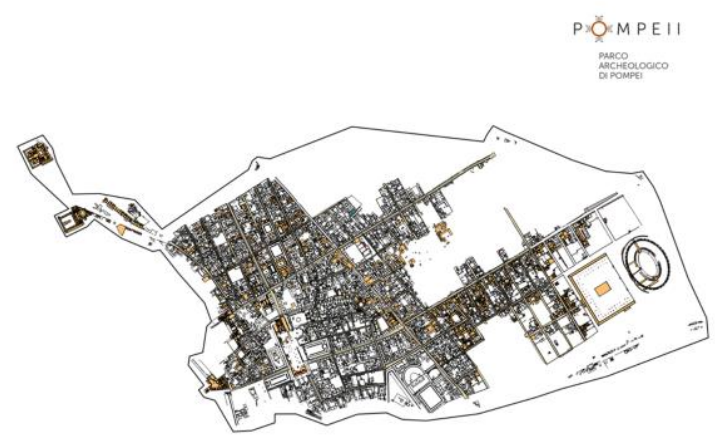

Figure 3. worksite area 


\subsection{The purpose of the GPP-IS}

The primary objective of the GPP-IS is to create a system that, based on a relational database linked to a georeferenced map, is to support the management of the 'planned maintenance' process, as defined by the Code of Cultural Cultural Heritage and by Art. 240 of the Regulation for the LL.PP. concerning cultural heritage assets (Legislative Decree 207/2010). The implementation of the planned maintenance aims to prevent degradation and monitor risk conditions, prioritising ordinary maintenance activities over restoration activities which intervene on already altered situations, and which are irremediably compromised by the loss of historical elements and evidence. A second, simultaneous objective is added to the primary objective, namely to constitute a body of knowledge of the ancient city, not only to be used for primary conservation purposes, but also for scientific and archaeological investigation, which Italian and international university missions, as well as the Superintendency, have been contributing to for decades.

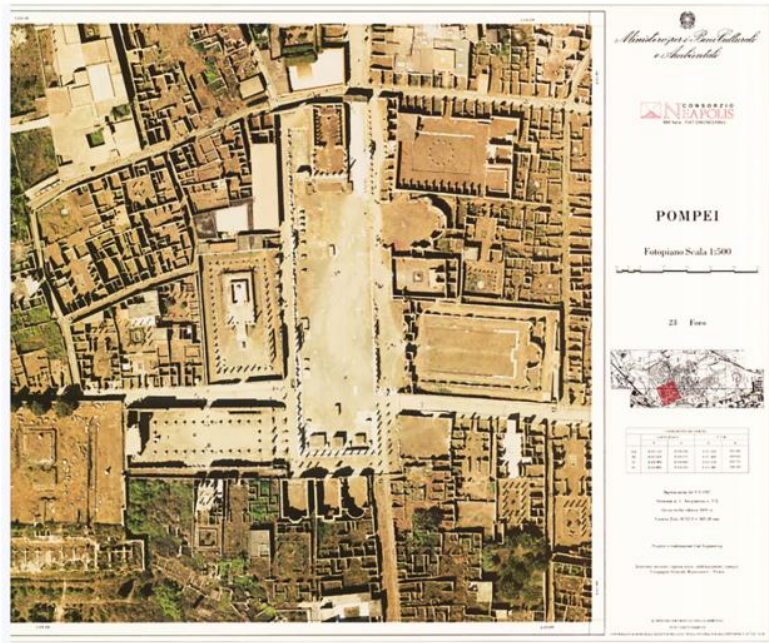

Figure 4. photopiano Pompei

Furthermore, the creation of a coordinated and homogenous set of data will allow us to have a reservoir of useful information to design and create development, diffusion and didactic projects, based on contents which hold recognised and scientific validity. The system structured in this way becomes the basis for the collection and homogenisation of previous data, as well as an instrument for recording information on archaeological and conservation aspects of the entire context of the ancient city of Pompeii, which are being implemented within the same project.

In practice, there are already WebGIS tools set up which allow the analysis of the state of conservation and the support of routine and extraordinary maintenance planning. The tools are based on attributes, spatial research and thematisation.

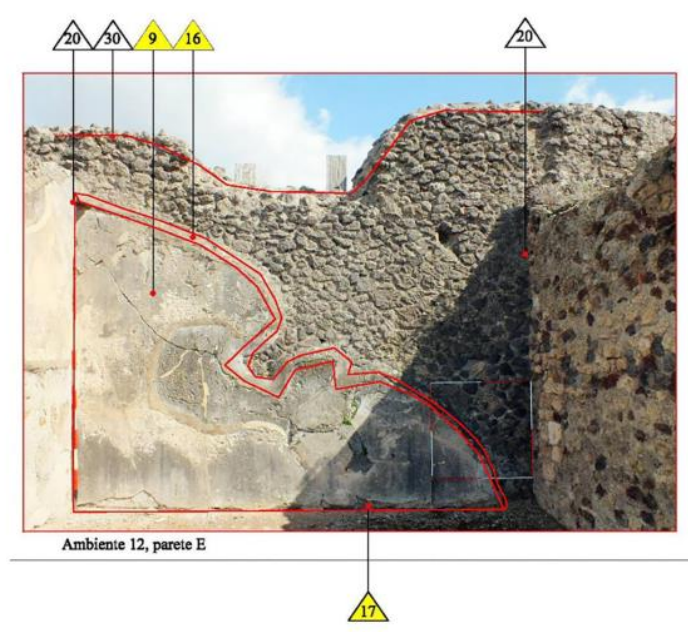

Figure 5. illustrative photo of mapping on a straightened photo

\subsection{GIS in archaeology:}

It is evident that GIS software has become a useful tool for the management of archaeological information, thanks to its ability to manage geographical, alphanumeric, cartographic and photographic data. In the academic field the use of this software has by now become habitual, but development possibilities are still numerous and under used. The use of these systems is still quite rare within the administration of Italian cultural heritage. The Pompeii IS is one of the largest and most complex Information Systems on the national archaeological scene, both in terms of site content and importance.

In the field of archaeology, it is essential that data is collected and organised in an organic manner right from the beginning and thus, having appropriately structured GIS tools available gives us the possibility to constantly check data, improving its quality and preventing information loss. Data management takes place through the rigorous and well organised compilation of the database where all the data is collected.

Additionally, in archaeology, the interpretation of data changes with the progress of excavation or in-depth analysis. The organisation of data in a GIS is dynamic by nature, as it changes over time, and can be updated when study and archaeological analyses allow the enhancing of knowledge, modifying already acquired elements if necessary, or the information connected to it.

The Pompeii SI is, therefore, not only useful for containing all of the data of the Ancient City in a structured and efficient way, but, thanks to possibilities now provided by the Web, it has become an exceptional carrier for sharing the immense wealth of information.

\subsection{Cultural Heritage will become a Common Good.}

Moreover, given the ever-increasing production of digital material courtesy of modern (graphic, photographic, 3D, vector, laserscanner, etc.) technology it should be noted that today, only using textual publication is no longer sufficient to illustrate the great wealth of archaeological information. These new indispensable systems which are available today give us the opportunity to consult, in a controlled way, all the data (and information) produced during even recent works of archaeological stratigraphic excavation. Technology is 
constantly evolving, and this should trigger a constant increase in information that the archaeologist is able to acquire. The Pompeii IS is structured and now ready to give these answers.

\subsection{Decision support}

The Pompeii IS, with its consultation/interrogation and also with its own geoprocessing ${ }^{3}$ criteria becomes a multi-criteria decision-making method which integrates archaeological data and environment factors, as well as geospatial analyses and predictive models.

By integrating the geographical component with the GIS, a Spatial Decision Support System $\left(\mathrm{SDSS}^{4}\right)$ is utilised for complex territorial analysis. They are not simply questions aimed at monitoring single information aspects, but rather summarising frameworks on aspects which have a strong impact on overall investment policies, and on the strategies that can be undertaken as well as the management of subsequent 'feedback': priority in maintenance operations, and the priority, sequence and completion of excavations; opening to the public of areas or parts of secured areas, etc.

Decision support, with the application of SDSS' own functions and the Pompeii IS consultation takes place with the quick and versatile extraction of information from a significant amount amount of data, and provides territorial information panels in the form of reports, thematic mates and presentations supported by GISes, even for requests of significant complexity.

An SSD, using various tools, allows us to increase the effectiveness of analyses, as it is able to generate a set of alternative options that the decision maker can evaluate.

Alternative options, prefigured in this way, can be directly taken into consideration, or used to hone analyses. Thus, the decision maker has the opportunity to have full control of the decisionmaking process, but also to correctly structure the process itself.

To summarise, by appropriately using the GIS equipment which the Pompeii IS consists of - it is possible to define the study of the problem, and provide interactive assistance to decision makers in order to solve structured and complex problems.

Only with the use of these state-of-the-art tools, interacting with the Pompeii IS, can we give swift answers to the city of Pompeii, which is the object of the world's attention, and is unanimously defined today as "the most alive of dead cities"...

\footnotetext{
${ }^{3}$ Geoprocessing is the name given to a family of GIS operations used for the manipulation of spatial data, generally implemented within more complex Spatial Analysis. In particular, when performing a geoprocessing operation, a set of spatial data is processed to generate one or more new outputs. The most common geoprocessing operations are: overlays, intersections between polygons, buffers, centroid calculation and more.

${ }^{4}$ According to Armstrong \& Densham (1990) an SDSS consists of 5 integrated modules:

1. Data Base Management System - DBMS

2. Applications for spatial analysis

3. ModelBase Management System

4. Report Generator

5. Graphic interface - GUI
}

\section{REFERENCES}

Michael Worboys \& Matt Duckham, GIS: a computing perspective, Boca Raton, London, New York, Washington, CRC Press, 2004, ISBN 978-0-203-48155-4.

Technical Annex "Sistema Informativo Grande Progetto Pompei" Ministry of Cultural Heritage and Activities Directorate General for Antiquities - Computer Design developed by: Maurizio D’Aristotile (MiPAAF) Stefano Frasca (INVITALIA) et al.

Che cos'è un GIS archeologico? Riflessioni a margine di alcune esperienze sul campo Alberto Monti Storicamente, 2 (2006). ISSN: 1825-411X. Art. no. 32. DOI: 10.1473/stor 306

Cattani M., 2008, Il percorso della conoscenza: applicazioni in archeologia tra ricerca e divulgazione, in Giuliano De Felice, Maria Giuseppina Sibilano, Giuliano Volpe (ed.) Information Technology and the method of Stratigraphy, Proceedings of the Workshop (Foggia 6-7th June 2008), Bari. 